\title{
Vigorous physical activity and carotid distensibility in young and mid-aged adults
}

\author{
Quan L Huynh $^{1}$, Christopher L Blizzard ${ }^{1}$, Olli Raitakari², James E Sharman ${ }^{1}$, Costan G Magnussen ${ }^{1,2}$, \\ Terence Dwyer $^{1,3}$, Markus Juonala ${ }^{2}$, Mika Kähönen ${ }^{4}$ and Alison J Venn ${ }^{1}$
}

\begin{abstract}
Although physical activity (PA) improves arterial distensibility, it is unclear which type of activity is most beneficial. We aimed to examine the association of different types of PA with carotid distensibility (CD) and the mechanisms involved. Data included 4503 Australians and Finns aged 26-45 years. Physical activity was measured by pedometers and was self-reported. CD was measured using ultrasound. Other measurements included resting heart rate (RHR), cardiorespiratory fitness (CRF), blood pressure, biomarkers and anthropometry. Steps/day were correlated with RHR (Australian men $r=-0.10$, women $r=-0.14$; Finnish men $r=-0.15$, women $r=-0.11 ; P<0.01$ ), CRF and biochemical markers, but not with CD. Self-reported vigorous leisure-time activity was more strongly correlated with RHR (Australian men $r=-0.23$, women $r=-0.19$; Finnish men $r=-0.20$, women $r=-0.13 ; P<0.001$ ) and CRF, and was correlated with CD (Australian men $r=0.07$; Finnish men $r=0.07$, women $r=0.08 ; P<0.05$ ). This relationship of vigorous leisure-time activity with $C D$ was mediated by RHR independently of potential confounders. In summary, vigorous leisure-time PA but not total or less intensive PA was associated with arterial distensibility in young to mid-aged adults. Promotion of vigorous PA is therefore recommended among this population. RHR was a key intermediary factor explaining the relationship between vigorous PA and arterial distensibility.

Hypertension Research (2015) 38, 355-360 doi:10.1038/hr.2015.9; published online 19 February 2015
\end{abstract}

Keywords: artery; compliance; exercise; heart rate; lifestyle; population

\section{INTRODUCTION}

Large arteries such as the carotid and aorta stiffen with age even in healthy individuals. ${ }^{1}$ Decreased distensibility (or increased stiffness) of large arteries can independently predict cardiovascular events and all-cause mortality, ${ }^{2-4}$ and is one of the most important contributors to the increased cardiovascular risk with aging. ${ }^{5}$ Thus, any lifestyle factors that can delay this process hold promise for reducing age-associated cardiovascular disease.

Intervention studies have reported light-to-moderate physical activity (PA) to increase arterial distensibility among older adults. ${ }^{6,7}$ However, it is unclear whether these results can be extrapolated to younger adults and there is limited information on the type of PA that exerts the most beneficial effect on arterial distensibility. Current guidelines ${ }^{8}$ reflect the thinking that reduction in cardiovascular risks can be achieved by participating in either vigorous PA or light-tomoderate PA (with longer time required). Walking, assessed by questionnaire or by motion sensors such as pedometers or accelerometers, has been shown to have a range of cardio-metabolic benefits $^{9-11}$ but its association with arterial distensibility is less clear. Recent evidence suggests that vigorous PA may provide cardioprotective benefits that are beyond those achieved through light-to-moderate
$\mathrm{PA}^{12,13}$ but associations of objectively measured steps/day with arterial distensibility have not been previously investigated. Clarifying this matter is important for informing advice on PA to promote cardiovascular health.

In previously published work, we found resting heart rate (RHR) to be a key intermediary factor in the positive relationship between cardiorespiratory fitness (CRF) and carotid distensibility (CD). ${ }^{14}$ In this study, using data on 4503 young to mid-aged adults from two large population-based cohorts in Australia and Finland, we examined the relationship of different types of PA, including pedometer measured steps/day, with $\mathrm{CD}$ and, for comparison, other cardiovascular risk factors. We sought to determine whether the association varied by type of PA and aimed to investigate whether RHR is also a key intermediary factor in this relationship.

\section{MATERIALS AND METHODS}

This study included data from two large population-based prospective cohort studies in Australia and Finland. Each study was approved by local ethics committees. All participants provided written informed consent.

${ }^{1}$ Menzies Research Institute Tasmania, University of Tasmania, Hobart, Australia; ${ }^{2}$ Research Centre of Applied and Preventive Cardiovascular Medicine, University of Turku, Turku, Finland; ${ }^{3}$ Murdoch Childrens Research Institute, Melbourne, Australia and ${ }^{4}$ Department of Clinical Physiology, University of Tampere and Tampere University Hospital, Tampere, Finland

Correspondence: Dr QL Huynh, Menzies Research Institute Tasmania, University of Tasmania, 17 Liverpool Street (Private bag 23), Hobart TAS 7000, Australia.

E-mail: Iqhuynh@utas.edu.au

Received 26 July 2014; revised 9 December 2014; accepted 12 December 2014; published online 19 February 2015 


\section{Australia: the Childhood Determinants of Adult Health study}

Study population. The Childhood Determinants of Adult Health (CDAH) study collected baseline data in 1985 on a nationally-representative sample of 8498 Australian schoolchildren aged $7-15$ years. ${ }^{15}$ In this study, we included 2328 non-pregnant participants aged $26-36$ years ( $49.4 \%$ male) who attended one of 34 study clinics across Australia at follow-up during May 2004-May $2006 .{ }^{16}$ Of these participants, $1787(76.8 \%)$ had their arterial distensibility measured.

Physical activity. Daily steps were recorded using Yamax Digiwalker SW-200 pedometers for 7 days. ${ }^{9}$ Average steps per day was calculated for participants wearing pedometers at least $8 \mathrm{~h}$ per day for at least 4 days, ${ }^{17}$ consistent with other studies. ${ }^{18} \mathrm{PA}$ in previous week was self-reported using the International Physical Activity Questionnaire. ${ }^{19}$ Minutes per week spent on work-related, domestic and recreational PA at moderate and vigorous intensity was recorded together with time spent in active transport (classified as moderate intensity). These were summed to obtain total minutes of moderate-to-vigorous PA, and moderate and vigorous activities were weighted-by assigning metabolic equivalent of task (MET) values of four and eight respectively—to obtain total PA energy expenditure.

Arterial distensibility and blood pressure. End-systolic and end-diastolic diameters, and intima-media thickness of the left common carotid artery were measured using a portable Acuson Cypress (Siemens Medical Solutions USA Inc., Mountainview, CA, USA) platform with a $7.0 \mathrm{MHz}$ linear-array transducer by a single technician, following a standardized protocol. ${ }^{16,20}$ Before its inclusion in the CDAH study, the ultrasound measures derived from this portable Acuson Cypress were validated against those from a routinely-used clinic-based ultrasound machine like that used in the Cardiovascular Risk in Young Finns study (Acuson Sequoia 512, Siemens Medical Solutions USA Inc.). ${ }^{21}$ Brachial systolic blood pressure (SBP) and diastolic blood pressure (DBP) were measured during the ultrasound with a mean of two readings used in this study. ${ }^{16}$ The inverse of stiffness (CD) was calculated as follows: ${ }^{14,16}$

$\mathrm{CD}=\left(\left[D_{\mathrm{sbp}}-D_{\mathrm{dbp}}\right] / D_{\mathrm{dbp}}\right) /(\mathrm{SBP}-\mathrm{DBP})$

where $D_{\mathrm{sbp}}$ and $D_{\mathrm{dbp}}$ are the end-systolic and end-diastolic diameters.

Other cardiovascular risk factors. Body mass index (BMI) was calculated as weight $(\mathrm{kg}) /$ height $\left(\mathrm{m}^{2}\right)$. Mean arterial pressure $(\mathrm{MAP})$ was calculated as MAP $=$ $1 / 3 \mathrm{SBP}+2 / 3 \mathrm{DBP}$. RHR was measured while sitting after at least a 5-min rest using an Omron HEM907 Blood Pressure Monitor (Omron Corporation, Kyoto, Japan). Concentrations of high-density lipoprotein cholesterol (HDL-C), triglycerides, insulin and glucose were measured in 12-h overnight fasting blood samples. ${ }^{16}$ Low-density lipoprotein cholesterol (LDL-C) concentration was calculated using the Friedewald formula. ${ }^{22}$ Because the absolute workload achieved is partly a function of muscle mass, ${ }^{23}$ physical working capacity measured by a bicycle ergometer ${ }^{24}$ at a heart rate of 170 beats per minute was adjusted for lean body mass to create an index of CRF that is uncorrelated with lean body mass. This was previously described elsewhere. ${ }^{14}$ The first principal component ${ }^{25}$ of five measures of strength (left and right grip, shoulder push and pull and leg strength) was measured by appropriate dynamometers (Smedley's Dynamometer, TTM, Tokyo, Japan) and adjusted for body weight to create an index of muscular strength that is uncorrelated with weight. ${ }^{14}$ Data on socio-economic status, smoking and alcohol consumption were obtained by questionnaire. ${ }^{16}$

\section{Finland: the Cardiovascular risk in Young Finns study}

Study population. The Young Finns study collected baseline data in 1980 on 3596 Finnish children and adolescents aged 3-18 years. ${ }^{26}$ In this study, we included 2175 non-pregnant participants aged $30-45$ years ( $45.8 \%$ male) who attended a clinic in Finland during the follow-up in 2007. Of these participants, $2169(99.7 \%)$ had their arterial distensibility measured.

Physical activity. In 2007, participants of Young Finns study wore Omron Walking Style One (HJ-152 R-E) pedometers (Kyoto, Japan) to record daily steps for 7 days. ${ }^{27}$ Average steps per day was calculated for participants wearing pedometers at least $8 \mathrm{~h}$ per day for 4 days, consistent with other studies including CDAH. ${ }^{9,18}$ Leisure-time and transport PA were obtained by questionnaire, and a value of MET.hour per week was calculated based on these two subdomains of PA for Young Finns participants, which has been described elsewhere. ${ }^{28}$ Vigorous leisure-time PA per usual week was reported by choosing one of the six response categories: 'none', 'approximately $30 \mathrm{~min}$ ', ' $1 \mathrm{~h}$ ', ' $2-3 \mathrm{~h}$ ', ' $4-6 \mathrm{~h}$ ' and ' $7 \mathrm{~h}$ or more'.

Arterial distensibility and blood pressure. Using a similar standardized protocol as in $\mathrm{CDAH},{ }^{16,20}$ end-systolic and end-diastolic diameters and intima-media thickness were measured from the left common carotid artery by Sequoia 512 ultrasound mainframes (Acuson) with $13.0 \mathrm{MHz}$ linear-array transducers, together with concomitant brachial blood pressure. CD was calculated using the same formula as in $\mathrm{CDAH} .{ }^{20}$

Other cardiovascular risk factors. Using similar standardized protocols and formulas as in CDAH, BMI and MAP were calculated, RHR was measured while sitting after at least a 5-min rest, 12 -h overnight fasting concentrations of HDL-C, triglycerides, insulin and blood glucose were measured, ${ }^{20}$ LDL-C concentration was calculated using the Friedewald formula, ${ }^{22}$ and data on socio-economic status, smoking and alcohol consumption were obtained by a questionnaire. ${ }^{29} \mathrm{CRF}$ was objectively estimated in a random subsample of 538 participants $(47.8 \%$ male) by a bicycle ergometer using hypothetical maximal workload sustainable for 6 min measured on the basis of age, sex, height and weight, following a previously reported protocol. ${ }^{30}$ Because this measure of CRF was estimated based on height and weight, we did not further adjust for lean body mass.

\section{Statistical analyses}

For analyses, right-skewed outcome data (CD and other risk factors) were transformed before estimation of their means or linear regression. The regression estimates reported are in the original units of the outcomes for one unit increase in the study factor. ${ }^{31}$ In both cohorts, vigorous PA was defined as those that required hard physical effort and made the participants breathe much harder than normal. Participants of each sex in each sample were classified as spending no time, $<2 \mathrm{~h}$ per week, $2-3 \mathrm{~h}$ per week or $>3 \mathrm{~h}$ per week in vigorous leisure-time PA. For estimation of trend, these ordered levels were graded by consecutive integer scores. For estimation of relative risk, low arterial distensibility was defined by having $\mathrm{CD}<10$ th percentile specific for each sample, each sex and each year of age. Based on the concept of 'vascular age' for intima-media thickness, ${ }^{32}$ we used linear regression to estimate sampleand sex-specific rates of decreasing CD per year of age after adjusting for BMI, $\mathrm{PA}$, socio-economic status, smoking and alcohol consumption. We then calculated the mean difference in 'vascular age' of participants who spent at least $1 \mathrm{~h}$ per week in vigorous $\mathrm{PA}$, as in guidelines, ${ }^{8}$ compared with that of those who did not by dividing the difference in $\mathrm{CD}$ by the rate of decreasing $\mathrm{CD}$ per year of age. The percentage by which RHR explained the relationship of PA with CD was calculated by subtracting the direct effect (adjusting for RHR and age) from the total effect (adjusting for age only), and then dividing by the total effect. The percentage was set at $100 \%$ if adjusting for RHR reversed the sign of the association. Because of similar results in the two cohorts, we reported pooled data analyses in the tables for ease of interpretation. Cohort-specific results are shown as supplementary content.

\section{RESULTS}

Characteristics of participants in the two samples are shown in Table 1. On average, the Young Finns participants were 6 years older than the CDAH participants. Other than having lower RHR, the men in each sample had lower CD and greater BMI, MAP, insulin, glucose, LDL-C and triglycerides, and lower HDL-C, than the women (all $P<0.001)$. The risk factor profile of the Young Finns reflected their slightly older age. The pooled data reflected average values of the $\mathrm{CDAH}$ and Young Finns samples and were consistent with the participant characteristics in each sample.

CRF was positively correlated with steps/day (CDAH men $r=0.15$, women $r=0.21$; Young Finns men $r=0.33$, women $r=0.25$; all $P<0.001$ ), and was more strongly correlated with self-reported 
Table 1 Characteristics of participants in the two samples

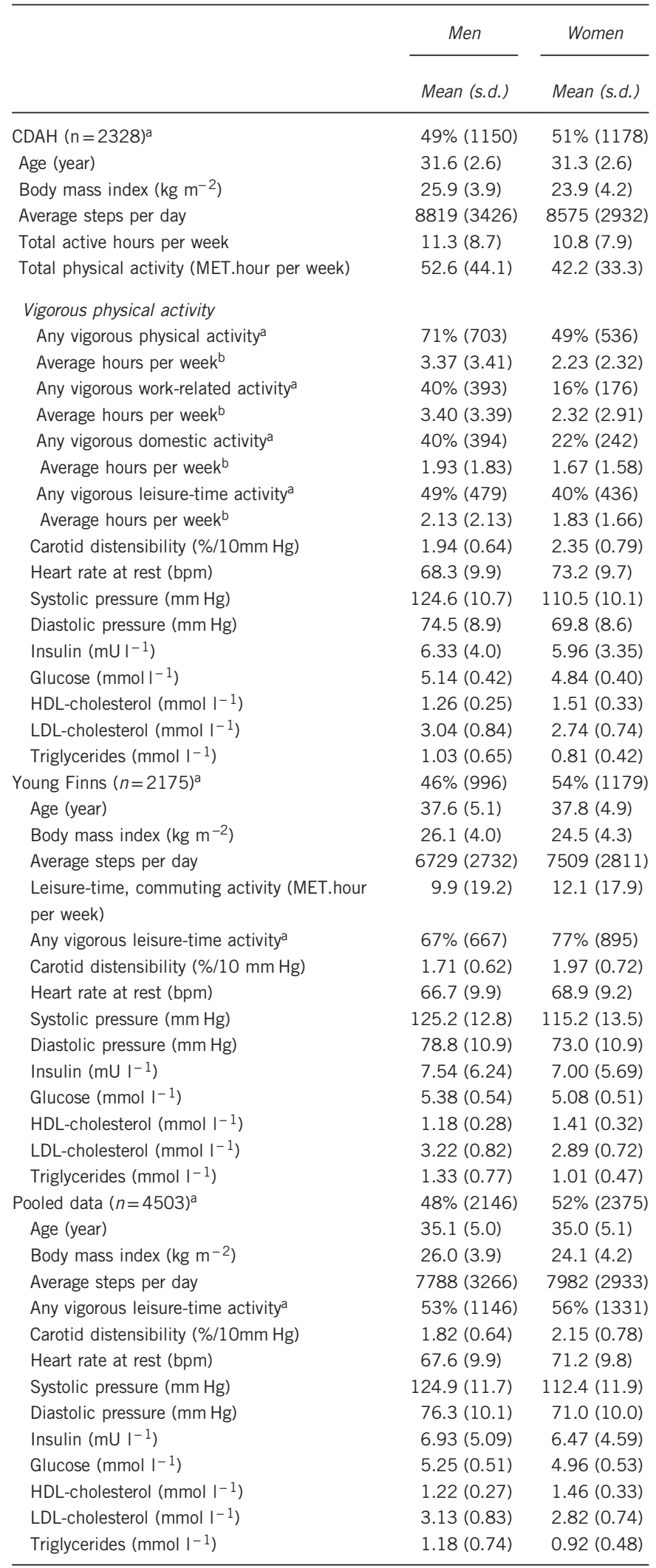

Abbreviations: $\mathrm{CDAH}$, the Childhood Determinants of Adult Health study; HDL, high-density lipoprotein; LDL, low-density lipoprotein; MET, metabolic equivalent of task; Young Finns, the Cardiovascular Risk in Young Finns study.

aData are percentage (number).

bData are reported only for participants who reported some vigorous physical activity of the corresponding subdomain. vigorous leisure-time PA (CDAH men $r=0.36$ women $r=0.35$, Young Finns men $r=0.41$ women $r=0.40$, all $P<0.001$ ).

Table 2 shows Spearman correlations of steps per day, and of self-reported $\mathrm{PA}$, with $\mathrm{CD}$ and other cardiovascular risk factors using pooled data. Cohort-specific correlations are reported in Supplementary Table 1 showing consistent results. Steps per day and total PA were negatively associated with RHR, insulin and triglycerides and positively associated with HDL-C, but not with CD. Among CDAH participants, self-reported time spent on walking was also not associated with $\mathrm{CD}$ (men $P=0.431$, women $P=0.968$ ). Although the associations of outcomes with total PA or total vigorous PA remained significant, they were largely reduced after adjustment for the vigorous leisure-time component of PA (not shown). Only vigorous leisuretime PA was positively associated with $\mathrm{CD}$.

Because only vigorous leisure-time PA was associated with $\mathrm{CD}$, we further investigated this association. Supplementary Table 2 shows that participants with greater vigorous leisure-time PA had greater mean values of $C D$. Whereas steps per day and self-reported walking and moderate PA (not shown) were not associated with $\mathrm{CD}$, participants spending at least $1 \mathrm{~h}$ per week in vigorous leisure-time PA had a lower vascular age of approximately 6.6 years (CDAH men), 1.0 year (CDAH women), 2.7 years (Young Finns men) and 3.2 years (Young Finns women) than those who did not. Based on consistent findings from parallel analyses, Table 3 shows pooled-data analyses adjusting for cohort and age, which presents associations of greater levels of vigorous leisure-time PA with lower risk of having low arterial distensibility (see Materials and Methods). In contrast, greater steps per day was not associated with risk of having low arterial distensibility (CDAH men $P=0.868$, women $P=0.254$; Young Finns men $P=0.883$, women $P=0.161$ ). Adjustment for socio-economic status, smoking and alcohol consumption, and muscular strength (CDAH only), did not change our findings (not shown).

Compared with BMI, MAP and blood biomarkers, RHR was most strongly correlated with $\mathrm{CD}$ (CDAH men $r=-0.33$ women $r=-0.24$; Young Finns men $r=-0.30$ women $r=-0.30 ; P<0.001$ ). To investigate the possible pathways by which participation in vigorous leisure-time PA may influence $\mathrm{CD}$, Table 4 presents the estimated effects of participation in vigorous leisure-time PA on CD with adjustment for RHR and other relevant factors. While adjustment for MAP, BMI or insulin, HDL-C, LDL-C and triglycerides reduced slight-to-moderately the estimated effect of vigorous leisure-time PA on $\mathrm{CD}$, adjustment for RHR substantially reduced this association by $100 \%$ among both men and women. Additional adjustment for MAP, BMI or blood biomarkers after adjustment for RHR provided small additional changes to the regression coefficients produced by adjustment for RHR. Cohort-specific analyses are reported in Supplementary Table 3 that shows similar results.

\section{DISCUSSION}

Our principal findings were as follows. First, vigorous leisure-time PA, but not total PA or less intensive forms of PA, was associated with greater CD. Second, our findings for the first time suggest that participation in vigorous leisure-time PA may be associated with $\mathrm{CD}$ (or delay in age-related arterial stiffening) by reducing RHR. Third, also for the first time, our findings from large population-based samples of younger adults showed that pedometer measured steps per day was not associated with $\mathrm{CD}$. These findings were independent of age, MAP, BMI, biochemical markers, socio-economic status, smoking and alcohol consumption.

Our study included only young to mid-aged adults who did not have the low levels of arterial distensibility typically found among 
Table 2 Spearman correlation of different measures of physical activity with carotid artery distensibility and other cardiovascular risk factors

\begin{tabular}{|c|c|c|c|c|c|c|c|c|}
\hline & $C D$ & $H R$ & $M A P$ & Insulin & Glucose & $H D L-C$ & $L D L-C$ & Triglycerides \\
\hline \multicolumn{9}{|l|}{ Men } \\
\hline Steps per day & 0.00 & $-0.12^{* * *}$ & -0.02 & $-0.14^{* * *}$ & -0.03 & $0.11^{* * *}$ & 0.01 & $-0.09 * * *$ \\
\hline Total PAa & -0.02 & $-0.05^{*}$ & 0.01 & $-0.15^{* * *}$ & 0.01 & $0.06^{* *}$ & 0.03 & 0.00 \\
\hline Leisure vig. $P A^{b}$ & $0.07 * *$ & $-0.21^{* * *}$ & -0.02 & $-0.15^{* * *}$ & $-0.10^{* * *}$ & $0.10^{* * *}$ & $-0.10^{* * *}$ & $-0.09 * * *$ \\
\hline \multicolumn{9}{|l|}{ Women } \\
\hline Steps per day & 0.03 & $-0.13^{* * *}$ & -0.00 & $-0.12^{* * *}$ & $-0.05^{*}$ & $0.10^{* * *}$ & $-0.05^{*}$ & $-0.10^{* * *}$ \\
\hline Total $\mathrm{PA}^{\mathrm{a}}$ & -0.01 & $-0.05^{*}$ & -0.01 & -0.03 & 0.01 & 0.02 & 0.03 & -0.01 \\
\hline Leisure vig. $\mathrm{PA}^{\mathrm{b}}$ & $0.05^{*}$ & $-0.17^{* * *}$ & 0.01 & $-0.11^{* * *}$ & $-0.05^{*}$ & $0.09^{* * *}$ & $-0.06^{* *}$ & $-0.08^{* * *}$ \\
\hline
\end{tabular}

Abbreviations: CD, carotid distensibility; HDL-C, high-density lipoprotein cholesterol; HR, heart rate; LDL-C, low-density lipoprotein cholesterol; MAP, mean arterial pressure; MET, metabolic equivalent of task; PA, physical activity.

All analyses were adjusted for cohort. ${ }^{*} P<0.05 ;{ }^{*} P<0.01 ;{ }^{* *} P<0.001$.

aTotal MET.hour per week.

bLevels of participation in vigorous leisure-time physical activities.

Table 3 Relative risks of having low arterial distensibility by levels of participation in vigorous leisure-time physical activity in the two samples (pooled-data analysis)

\begin{tabular}{|c|c|c|c|c|c|c|}
\hline & \multicolumn{3}{|c|}{ Men } & \multicolumn{3}{|c|}{ Women } \\
\hline & $n / N$ & $R R$ & $(95 \% \mathrm{Cl})$ & $n / N$ & $R R$ & $(95 \% \mathrm{Cl})$ \\
\hline \multicolumn{7}{|c|}{ Vigorous leisure-time $P A$} \\
\hline None & $93 / 744$ & 1.00 & Ref & $103 / 881$ & 1.00 & Ref \\
\hline Any & $83 / 1047$ & 0.62 & $(0.46,0.82)$ & $115 / 1284$ & 0.73 & $(0.56,0.96)$ \\
\hline \multicolumn{7}{|c|}{ Vigorous leisure-time PA } \\
\hline None & $93 / 744$ & 1.00 & Ref & $103 / 881$ & 1.00 & Ref \\
\hline$<2 \mathrm{~h}$ per week & $33 / 351$ & 0.73 & $(0.50,1.07)$ & $54 / 533$ & 0.83 & $(0.60,1.15)$ \\
\hline 2-3 h per week & $31 / 404$ & 0.60 & $(0.40,0.88)$ & $36 / 486$ & 0.60 & $(0.40,0.87)$ \\
\hline$>3 \mathrm{~h}$ per week & $19 / 292$ & 0.51 & $(0.32,0.83)$ & $25 / 265$ & 0.73 & $(0.50,1.06)$ \\
\hline & \multicolumn{3}{|c|}{$P_{\text {trend }}=0.003$} & \multicolumn{3}{|c|}{$P_{\text {trend }}=0.03$} \\
\hline
\end{tabular}

Abbreviations: RR, relative risk; PA, physical activity.

Low arterial distensibility was defined by having carotid distensibility $<10$ th percentile specific for each sample, each sex and each year of age.

All models were adjusted for age and cohort.

Table 4 Regression of carotid distensibility on levels of participation in vigorous physical activity during leisure time, with adjustment for heart rate and other relevant factors

\begin{tabular}{|c|c|c|}
\hline & \multicolumn{2}{|c|}{ Carotid distensibility (\%/10mm Hg) } \\
\hline & \multirow{2}{*}{$\begin{array}{c}\text { Men } \\
\beta(95 \% \mathrm{Cl})\end{array}$} & \multirow{2}{*}{$\begin{array}{c}\text { Women } \\
\beta(95 \% \mathrm{Cl})\end{array}$} \\
\hline & & \\
\hline Unadjusted & $0.40(0.14,0.66)$ & $0.41(0.09,0.73)$ \\
\hline \multicolumn{3}{|l|}{ Adjusted for } \\
\hline Age & $0.34(0.08,0.60)$ & $0.36(0.04,0.67)$ \\
\hline Age, MAP & $0.34(0.08,0.60)$ & $0.36(0.05,0.67)$ \\
\hline Age, BMI & $0.28(0.03,0.54)$ & $0.23(0.01,0.49)$ \\
\hline Age, Biomarkers ${ }^{a}$ & $0.28(0.02,0.54)$ & $0.21(0.00,0.40)$ \\
\hline Age, RHR & $-0.05(-0.30,0.20)$ & $-0.03(-0.34,0.27)$ \\
\hline Age, RHR, MAP & $-0.01(-0.26,0.24)$ & $0.01(-0.28,0.32)$ \\
\hline Age, RHR, BMI & $-0.07(-0.32,0.18)$ & $-0.15(-0.46,0.15)$ \\
\hline Age, RHR, Biomarkers ${ }^{a}$ & $-0.11(-0.37,0.14)$ & $-0.07(-0.38,0.23)$ \\
\hline \multicolumn{3}{|c|}{$\begin{array}{l}\text { Abbreviations: BMI, body mass index; CDAH, the Childhood Determinants of Adult Health study; } \\
\text { CI, confidence intervals; MAP, mean arterial pressure; HDL, low-density lipoprotein; LDL, low- } \\
\text { density lipoprotein; RHR, resting heart rate; Young Finns, the Cardiovascular Risk in Young } \\
\text { Finns study. } \\
\text { All analyses were adjusted for cohort. } \\
\text { alncluding adjustment for insulin, HDL-cholesterol, LDL-cholesterol and triglycerides. }\end{array}$} \\
\hline
\end{tabular}

older people. Light-to-moderate PA has been found to increase arterial distensibility among old adults, ${ }^{33}$ but more vigorous PA may be required to increase the higher levels of arterial distensibility among younger adults as shown in our study. This is consistent with a study of a small sample of young adults. ${ }^{13}$ The same logic may explain the weaker association found for CDAH women in our study. Women in this age range have higher $\mathrm{CD}$ than men. This was observed in both the CDAH and Young Finns samples, and also in other studies. ${ }^{34,35} \mathrm{In}$ our study, CDAH women had the highest mean level of CD compared with $\mathrm{CDAH}$ men and Young Finns men and women and, as shown in Supplementary Table 2, even CDAH women who did not do any vigorous leisure-time $\mathrm{PA}$ had greater $\mathrm{CD}$ than the $\mathrm{CDAH}$ men who spent more than $3 \mathrm{~h}$ per week engaged in vigorous leisure-time PA. Thus, any benefits for $\mathrm{CD}$ of participation in vigorous PA may have been marginal or undetectable.

Current recommendations for $\mathrm{PA}^{8}$ reflect the evidence that cardiovascular risk can be reduced by either vigorous PA or moderate PA but with longer time required for moderate PA to achieve the same benefits. In our study, the benefits of PA in respect of greater $\mathrm{CD}$ were confined to vigorous leisure-time PA, and associations with RHR and CRF were greater for this type of PA as well. Whereas light-tomoderate PA was not associated with greater $\mathrm{CD}$, participants who spent at least $1 \mathrm{~h}$ per week on vigorous $\mathrm{PA}$ as described by the guideline ${ }^{8}$ had younger vascular age than those who did not. Although 
our findings do not contradict the benefits of light-to-moderate PA such as walking for cardiovascular health, they suggest that an increase in arterial distensibility among young to mid-aged adults may be best achieved by participation in vigorous PA and, are consistent with other evidence of vigorous PA being associated with greater cardioprotective benefits. ${ }^{12}$

In previously published work using the Australian sample, ${ }^{14}$ we found a positive relationship of CRF with CD that was mediated by RHR. This finding was not due to RHR being a surrogate marker of CRF and was previously discussed. ${ }^{14}$ In this study, RHR consistently mediated the positive relationship of vigorous leisure-time PA with $\mathrm{CD}$ in each of the Australian and Finnish sample. Though not completely understood, high RHR may increase mechanical load on the arterial wall by exposure to higher mean pressure and increase cyclic shear stress by shortening the diastolic period. ${ }^{36}$ This might lead to greater arterial wall stiffness possibly by promoting vascular smooth muscle cell growth and collagen deposition. ${ }^{36}$ Using data from $\mathrm{CDAH}$, we found that stroke volume was negatively associated with RHR, but was not related to $\mathrm{CD}$. This again suggests that the lower arterial distensibility associated with high RHR may be due to remodelling of the arterial wall.

This study used two large population-based samples of adults in Australia and Finland on whom standardized measurements were made of an extensive range of study factors. Despite many cultural and environmental differences in the two countries, the very consistent results from the two populations strengthened the external validity of our findings. Using similar standardized protocols for physical measurements (including $\mathrm{CD}$ ) in the two cohorts was another strength of this study. Although transducers with different frequencies were used, the coefficients of variation between these methods were very similar, ${ }^{16}$ suggesting comparable accuracy. Combining data on both self-reported and objectively-measured PA helps to clarify the beneficial effects of PA at different intensities for cardiovascular health. The validity of self-reported PA in our study was confirmed by its strong association with objectively-measured CRF. We were able to somewhat differentiate endurance training from strength training, which may have adverse effects on arterial distensibility, ${ }^{37}$ by accounting for muscular strength (in the CDAH sample). A limitation of our study was the use of brachial, instead of carotid, pulse pressure to calculate CD. This is likely to have resulted in an underestimation of the reported association between RHR and CD because the most physically-active people (with higher CD and lower RHR) would also be more likely to have the greatest systolic and pulse pressure amplification (higher brachial compared with central systolic and pulse pressure). The higher estimated values for SBP may therefore underestimate the calculated CD. The cross-sectional design of this study, however, limits the causal inferences concerning the relationship of vigorous PA with $\mathrm{CD}$. We cannot rule out the possibility of reverse causation whereby participants with stiffer arteries might do less vigorous PA. However, our findings on these generally-healthy individuals who were unaware of their levels of arterial distensibility link well with previous findings to suggest that young and mid-aged adults may acquire additional benefits of increased arterial distensibility by participation in vigorous leisure-time PA; a benefit mediated via lower RHR.

In summary, our findings provide further evidence to support the recommendation of vigorous PA for cardiovascular health benefits beyond those achieved through light-to-moderate PA such as walking in young to mid-aged adults and, for the first time, suggest that participation in vigorous PA may increase arterial distensibility through lower RHR.

\section{CONFLICT OF INTEREST}

The authors declare no conflict of interest.

\section{ACKNOWLEDGEMENTS}

We gratefully acknowledge the contributions of all CDAH and Young Finns staff and volunteers, and the participants. The CDAH study was funded by grants from the Australian National Health and Medical Research Council, the Australian National Heart Foundation, the Tasmanian Community Fund and Veolia Environmental Services. We gratefully thank the CDAH study sponsors (Sanitarium Health Food Company, ASICS Oceania and Target Australia). The Young Finns study was funded by the Academy of Finland, the Social Insurance Institution of Finland, Turku University Foundation, Juho Vainio Foundation, Finnish Foundation of Cardiovascular Research, Tampere University Hospital, and the Finnish Cultural Foundation. Authors CLB, JES, CGM and AJV were supported by fellowships from the Australian National Health and Medical Research Council.

1 Avolio AP, Deng FQ, Li WQ, Luo YF, Huang ZD, Xing LF, O'Rourke MF. Effects of aging on arterial distensibility in populations with high and low prevalence of hypertension: comparison between urban and rural communities in China. Circulation 1985; 71: 202-210.

2 Blacher J, Pannier B, Guerin AP, Marchais SJ, Safar ME, London GM. Carotid arterial stiffness as a predictor of cardiovascular and all-cause mortality in end-stage renal disease. Hypertension 1998; 32: 570-574.

3 Barenbrock M, Kosch M, Joster E, Kisters K, Rahn KH, Hausberg M. Reduced arterial distensibility is a predictor of cardiovascular disease in patients after renal transplantation. J Hypertens 2002; 20: 79-84.

4 Vlachopoulos C, Aznaouridis K, Stefanadis C. Prediction of cardiovascular events and all-cause mortality with arterial stiffness: a systematic review and meta-analysis. J Am Coll Cardiol 2010; 55: 1318-1327.

5 Lakatta EG, Levy D. Arterial and cardiac aging: major shareholders in cardiovascular disease enterprises: Part I: aging arteries: a 'set up' for vascular disease. Circulation 2003; 107: 139-146.

6 Tanaka H, Dinenno FA, Monahan KD, Clevenger CM, DeSouza CA, Aging Seals DR. habitual exercise, and dynamic arterial compliance. Circulation 2000; 102: 1270-1275.

7 Sugawara J, Otsuki T, Tanabe T, Hayashi K, Maeda S, Matsuda M. Physical activity duration, intensity, and arterial stiffening in postmenopausal women. Am J Hypertens 2006; 19: 1032-1036.

8 Haskell WL, Lee IM, Pate RR, Powell KE, Blair SN, Franklin BA, Macera CA, Heath GW, Thompson PD, Bauman A. Physical activity and public health: updated recommendation for adults from the American College of Sports Medicine and the American Heart Association. Circulation 2007; 116: 1081-1093.

9 Cleland VJ, Schmidt MD, Salmon J, Dwyer T, Venn A. Correlates of pedometermeasured and self-reported physical activity among young Australian adults. J Sci Med Sport 2011; 14: 496-503.

10 Dwyer T, Ponsonby AL, Ukoumunne OC, Pezic A, Venn A, Dunstan D, Barr E, Blair S, Cochrane J, Zimmet $P$, Shaw J. Association of change in daily step count over five years with insulin sensitivity and adiposity: population based cohort study. BMJ 2011; 342: c7249.

11 Schmidt MD, Cleland VJ, Shaw K, Dwyer T, Venn AJ. Cardiometabolic risk in younger and older adults across an index of ambulatory activity. Am J Prev Med 2009; 37 . 278-284.

12 Swain DP, Franklin BA. Comparison of cardioprotective benefits of vigorous versus moderate intensity aerobic exercise. Am J Cardiol 2006; 97: 141-147.

13 van de Laar RJ, Ferreira I, van Mechelen W, Prins MH, Twisk JW, Stehouwer CD. Lifetime vigorous but not light-to-moderate habitual physical activity impacts favorably on carotid stiffness in young adults: the amsterdam growth and health longitudinal study. Hypertension 2010; 55: 33-39.

14 Quan HL, Blizzard CL, Sharman JE, Magnussen CG, Dwyer T, Raitakari O, Cheung M, Venn AJ. Resting heart rate and the association of physical fitness with carotid artery stiffness. Am J Hypertens 2014; 27: 65-71.

15 Dwyer T, Gibbons LE. The Australian Schools Health and Fitness Survey. Physical fitness related to blood pressure but not lipoproteins. Circulation 1994; 89: 1539-1544.

16 Huynh Q, Blizzard L, Sharman J, Magnussen C, Schmidt M, Dwyer T, Venn A. Relative contributions of adiposity in childhood and adulthood to vascular health of young adults. Atherosclerosis 2013; 228: 259-264.

17 Schmidt MD, Blizzard CL, Venn AJ, Cochrane JA, Dwyer T. Practical considerations when using pedometers to assess physical activity in population studies: lessons from the Burnie Take Heart Study. Res Q Exerc Sport 2007; 78: 162-170.

18 Tudor-Locke CE, Myers AM. Methodological considerations for researchers and practitioners using pedometers to measure physical (ambulatory) activity. Res Q Exerc Sport 2001; 72: 1-12. 
19 Craig CL, Marshall AL, Sjostrom M, Bauman AE, Booth ML, Ainsworth BE, Pratt M, Ekelund $U$, Yngve A, Sallis JF, Oja P. International physical activity questionnaire 12-country reliability and validity. Med Sci Sports Exerc 2003; 35: 1381-1395.

20 Juonala M, Jarvisalo MJ, Maki-Torkko N, Kahonen M, Viikari JS, Raitakari OT. Risk factors identified in childhood and decreased carotid artery elasticity in adulthood: the Cardiovascular Risk in Young Finns Study. Circulation 2005; 112: 1486-1493.

21 Magnussen CG, Fryer J, Venn A, Laakkonen M, Raitakari OT. Evaluating the use of a portable ultrasound machine to quantify intima-media thickness and flow-mediated dilation: agreement between measurements from two ultrasound machines. Ultrasound Med Biol 2006; 32: 1323-1329.

22 Friedewald WT, Levy RI, Fredrickson DS. Estimation of the concentration of low-density lipoprotein cholesterol in plasma, without use of the preparative ultracentrifuge. Clin Chem 1972; 18: 499-502.

23 Buskirk E, Taylor HL. Maximal oxygen intake and its relation to body composition, with special reference to chronic physical activity and obesity. J Appl Physiol 1957; 11: 72-78.

24 Withers RT, Davies GJ, Crouch RG. A comparison of three W170 protocols. Eur J Appl Physiol Occup Physiol 1977; 37: 123-128.

25 Hotelling $\mathrm{H}$. Analysis of a complex of statistical variables into principal components. $J$ Educ Psychol 1933; 24: 417-441.

26 Koskinen J, Magnussen CG, Taittonen L, Rasanen L, Mikkila V, Laitinen T, Ronnemaa T, Kahonen M, Viikari JS, Raitakari OT, Juonala M. Arterial structure and function after recovery from the metabolic syndrome: the cardiovascular risk in Young Finns Study. Circulation 2010; 121: 392-400.

27 Hirvensalo M, Telama R, Schmidt MD, Tammelin TH, Xiaolin Y, Magnussen CG, Vkari JS, Raitakari OT. Daily steps among Finnish adults: variation by age, sex, and socioeconomic position. Scand J Public Health 2011; 39: 669-677.

28 Mansikkaniemi K, Juonala M, Taimela S, Hirvensalo M, Telama R, Huupponen R, Saarikoski L, Hurme M, Mallat Z, Benessiano J, Jula A, Taittonen L, Marniemi J, Kahonen M, Lehtimaki T, Ronnemaa T, Viikari J, Raitakari OT. Cross-sectional associations between physical activity and selected coronary heart disease risk factors in young adults. The Cardiovascular Risk in Young Finns Study. Ann Med 2012; 44 733-744.

29 Aatola H, Koivistoinen T, Hutri-Kahonen N, Juonala M, Mikkila V, Lehtimaki T, Viikari JS, Raitakari OT, Kahonen M. Lifetime fruit and vegetable consumption and arteria pulse wave velocity in adulthood: the Cardiovascular Risk in Young Finns Study. Circulation 2010; 122: 2521-2528.

30 Arstila M, Impivaara O, Maki J. New ergometric reference values for clinical exercise tests. Scand J Clin Lab Invest 1990; 50: 747-755.

31 Hosmer D, Blizzard L. Estimating effects for non-linearly scaled covariates in regression models Part I - Linear link function models. Australas Epidemiol 2004; 11: 40-47.

32 Stein JH, Fraizer MC, Aeschlimann SE, Nelson-Worel J, McBride PE, Douglas PS. Vascular age: integrating carotid intima-media thickness measurements with global coronary risk assessment. Clin Cardiol 2004; 27: 388-392.

33 Gando Y, Yamamoto K, Murakami H, Ohmori Y, Kawakami R, Sanada K, Higuchi M, Tabata I, Miyachi M. Longer time spent in light physical activity is associated with reduced arterial stiffness in older adults. Hypertension 2010; 56: 540-546.

34 Ferreira I, Twisk JW, van Mechelen W, Kemper HC, Seidell JC, Stehouwer CD. Current and adolescent body fatness and fat distribution: relationships with carotid intimamedia thickness and large artery stiffness at the age of 36 years. J Hypertens 2004; 22: 145-155.

35 Doonan RJ, Mutter A, Egiziano G, Gomez YH, Daskalopoulou SS. Differences in arteria stiffness at rest and after acute exercise between young men and women. Hypertens Res 2013; 36: 226-231.

36 Giannoglou GD, Chatzizisis YS, Zamboulis C, Parcharidis GE, Mikhailidis DP, Louridas GE. Elevated heart rate and atherosclerosis: an overview of the pathogenetic mechanisms. Int J Cardiol 2008; 126: 302-312.

37 Miyachi M, Kawano H, Sugawara J, Takahashi K, Hayashi K, Yamazaki K, Tabata I, Tanaka $\mathrm{H}$. Unfavorable effects of resistance training on central arterial compliance: a randomized intervention study. Circulation 2004; 110: 2858-2863.

Supplementary Information accompanies the paper on Hypertension Research website (http://www.nature.com/hr) 